\section{$L A-13661$}

Approved for public release; distribution is unlimited.

\section{EOS of a Material Mixture in}

\section{Pressure Equilibrium}

\section{Charles W. Cranfill}

\section{Los Alamos \\ NATIONAL LABOAATORY}

Los Alamos National Laboratory is operated by the University of California for the United States Department of Energy under contract W-7405-ENG-36. 


\section{DISCLAIMER}

This report was prepared as an account of work sponsored by an agency of the United States Government. Neither the United States Government nor any agency thereof, nor any of their employees, make any warranty, express or implied, or assumes any legal liability or responsibility for the accuracy, completeness, or usefulness of any information, apparatus, product, or process disciosed, or represents that its use would not infringe privately owned rights. Reference herein to any specific commercial product, process, or service by trade name, trademark, manufacturer, or otherwise does not necessarily constitute or imply its endorsement, recommendation, or favoring by the United States Government or any agency thereof. The views and opinions of authors expressed herein do not necessarily state or reflect those of the United States Government or any agency thereof. 


\section{DISCLAIMER}

Portions of this document may be illegible in electronic image products. Images are produced from the best available original document. 


\title{
EOS OF A MATERIAL MIXTURE IN PRESSURE EQUILIBRIUM
}

\author{
by
}

\author{
Charles W. Cranfill
}

\begin{abstract}
A procedure is presented for calculating the equation of state (EOS) of a material mixture whose constituents are in pressure equilibrium but not necessarily in temperature equilibrium. A Newton-Raphson iteration is performed to determine the constituent partial volumes and energies that give equal partial pressures subject to the constraints that the total volume and energy of the mixture are specified. During each iteration, the changes in EOS quantities are chosen to be fixed (but arbitrary) linear combinations of their temperature-equilibrium and isentropic values, so those two extremes can be reproduced as special cases. The changes in the constituent partial volumes and energies are limited to prevent the Newton-Raphson iteration from diverging.
\end{abstract}

\section{Introduction.}

In fluid-dynamical systems containing multiple materials, the problem of calculating the equation of state (EOS) of a material mixture often arises. Various relaxation processes, such as rarefactionwave propagation, thermal conduction, and molecular diffusion, tend to drive the constituents of a mixture toward pressure and temperature equilibrium, a condition which minimizes the Gibbs free energy and simplifies the calculation of the EOS. However, the relaxation rates depend upon the degree of contact of the mixture constituents, i.e. the fractions of atomic versus chunk mix and the distribution of chunk sizes and shapes, so a dynamical mixture can fail to achieve complete equilibrium. Thus the calculation of the EOS of a nonequilibrium mixture is sometimes required.

This report describes a procedure for calculating the EOS of a material mixture whose constituents are in pressure equilibrium but not necessarily in temperature equilibrium. A NewtonRaphson iteration ${ }^{1}$ is performed to determine the constituent partial volumes and energies that give equal partial pressures subject to the constraints that the total volume and energy of the mixture are specified. During each iteration, the changes in EOS quantities are chosen to be fixed (but arbitrary) linear combinations of their temperature-equilibrium and isentropic values, so those two extremes can be reproduced as special cases. Enforcing the constant total volume and energy constraints at every iteration should enhance the efficiency of the procedure, but the convergence of the Newton-Raphson iteration still requires that the initial guess be sufficiently close to the final solution. Since such an accurate initial guess cannot be guaranteed, the changes in the constituent partial volumes and energies are limited to prevent the Newton-Raphson iteration from diverging.

The system to be considered is a material mixture whose constituents (labeled with the subscript index $j$ ) have masses $M_{j}$, initial specific volumes $v_{j}^{(0)}$, initial specific energies $e_{j}^{(0)}$, and EOS functions 
for pressure $P_{j}(v, e)$ and temperature $T_{j}(v, e)$. EOS functions for specific entropy $s_{j}(v, e)$ are also used in the following analysis but never need to be evaluated explicitly. During the iterations (labeled with the superscript index $i$ ) to determine the specific volumes and energies that produce equal partial pressures, the initial total volume and energy of the mixture are preserved:

$$
\sum_{j} M_{j} v_{j}^{(i)}=\sum_{j} M_{j} v_{j}^{(0)} \equiv V, \quad \sum_{j} M_{j} e_{j}^{(i)}=\sum_{j} M_{j} e_{j}^{(0)} \equiv E
$$

In practice, the complexity of EOS functions often requires that they be presented in tabular rather than analytic form. The values of the dependent variables for specified values of the independent variables are then determined by interpolation among the tabulated values. The Sesame EOS Data Library, ${ }^{2,3}$ created and maintained by $\mathrm{T}$ Division at the Los Alamos National Laboratory (LANL), is such a tabulation of EOS functions, giving pressure $P$ and specific energy $e$ as functions of mass density $\rho=1 / v$ and temperature $T$ for a wide range of materials. The EOSPAC subroutine package,${ }^{4}$ created and maintained by X Division at LANL, accesses and interpolates the Sesame EOS data tables to calculate values for EOS functions and their first partial derivatives. EOSPAC also provides the option to invert the Sesame EOS data tables to replace temperature $T$ with specific energy $e$ as the second independent variable. The MIXPAC subroutine package, ${ }^{5}$ also created and maintained by X Division at LANL, uses EOSPAC along with a Newton-Raphson iteration to calculate the EOS of a material mixture whose constituents are in pressure and temperature equilibrium. The purpose of this report is to generalize the MIXPAC procedure to allow the constituents to be out of temperature equilibrium.

The remainder of this report is divided into sections presenting some useful thermodynamic relations, the temperature-equilibrium and isentropic cases, the generalized procedure, and some conclusions. An appendix discusses preventing the Newton-Raphson iteration from diverging.

\section{Thermodynamic Relations.}

This section lists some useful thermodynamic relations among small changes in and first partial derivatives of various EOS functions. These relations are consequences of the first law of thermodynamics combined with first-order Taylor-series expansions and the chain rule for partial differentiation applied to functions of two independent variables. Five EOS functions are of interest: the two intensive quantities pressure $P$ and temperature $T$, and the three extensive quantities specific volume $v$, specific energy $e$, and specific entropy $s$. It is assumed that each of these quantities can be expressed as a single-valued function of any two of the others, and small changes in the quantities are represented by the symbol $\delta$.

The most convenient choice for the two independent variables depends upon the intended application: $(v, e)$ is best for preserving total volume and energy; $(P, T)$ is best for forcing pressure and temperature equilibrium; $(P, s)$ is best for forcing isentropic pressure equilibrium; $(v, s)$ is best for applying the first law of thermodynamics; and $(v, T)$ is best for using the Sesame EOS data tables (although $(v, e)$ is best for using the EOSPAC-inverted data tables). The following thermodynamic relations allow easy transformations among these independent-variable choices. Note that the relations among first partial derivatives are exact consequences of the chain rule for partial differentiation, but the relations among changes in the EOS quantities are first-order approximations that become exact only when those changes are infinitesimal. 


$$
\begin{aligned}
& \delta v=\delta P(\partial v / \partial P)_{T}+\delta T(\partial v / \partial T)_{P}=\delta P(\partial v / \partial P)_{s}+\delta s(\partial v / \partial s)_{P} \\
& \delta e=\delta P(\partial e / \partial P)_{T}+\delta T(\partial e / \partial T)_{P}=\delta P(\partial e / \partial P)_{s}+\delta s(\partial e / \partial s)_{P}=-P \delta v+T \delta s \\
& \delta s=\delta P(\partial s / \partial P)_{T}+\delta T(\partial s / \partial T)_{P}=(P / T) \delta v+(1 / T) \delta e \\
& (\partial e / \partial v)_{s}=-P=-T(\partial s / \partial v)_{e}=(\partial e / \partial v)_{T}-T(\partial s / \partial v)_{T} \\
& (\partial e / \partial s)_{v}=T=P /(\partial s / \partial v)_{e}=(\partial e / \partial T)_{v} /(\partial s / \partial T)_{v} \\
& (\partial T / \partial v)_{s}=-(\partial P / \partial s)_{v}=-T(\partial P / \partial e)_{v}=-T(\partial P / \partial T)_{v} /(\partial e / \partial T)_{v} \\
& (\partial s / \partial v)_{T}=(\partial P / \partial T)_{v}=(\partial P / \partial e)_{v} /(\partial T / \partial e)_{v} \\
& (\partial s / \partial P)_{T}=(P / T)(\partial v / \partial P)_{T}+(1 / T)(\partial e / \partial P)_{T} \\
& (\partial s / \partial T)_{P}=(P / T)(\partial v / \partial T)_{P}+(1 / T)(\partial e / \partial T)_{P} \\
& \partial(P, T) / \partial(v, e) \equiv(\partial P / \partial v)_{e}(\partial T / \partial e)_{v}-(\partial T / \partial v)_{e}(\partial P / \partial e)_{v} \equiv-\partial(T, P) / \partial(v, e) \\
& \partial(P, e) / \partial(v, T) \equiv(\partial P / \partial v)_{T}(\partial e / \partial T)_{v}-(\partial e / \partial v)_{T}(\partial P / \partial T)_{v} \equiv-\partial(e, P) / \partial(v, T) \\
& (\partial v / \partial P)_{T}=(\partial T / \partial e)_{v} /[\partial(P, T) / \partial(v, e)]=1 /(\partial P / \partial v)_{T} \\
& (\partial v / \partial T)_{P}=-(\partial P / \partial e)_{v} /[\partial(P, T) / \partial(v, e)]=-(\partial P / \partial T)_{v} /(\partial P / \partial v)_{T} \\
& (\partial v / \partial P)_{s}=1 /\left[(\partial P / \partial v)_{e}-P(\partial P / \partial e)_{v}\right]=(\partial e / \partial T)_{v} /\left[\partial(P, e) / \partial(v, T)-P(\partial P / \partial T)_{v}\right] \\
& (\partial v / \partial s)_{P}=-T(\partial P / \partial e)_{v}(\partial v / \partial P)_{s}=-T\left[(\partial P / \partial T)_{v} /(\partial e / \partial T)_{v}\right](\partial v / \partial P)_{s} \\
& (\partial e / \partial P)_{T}=-(\partial T / \partial v)_{e} /[\partial(P, T) / \partial(v, e)]=(\partial e / \partial v)_{T} /(\partial P / \partial v)_{T} \\
& (\partial e / \partial T)_{P}=(\partial P / \partial v)_{e} /[\partial(P, T) / \partial(v, e)]=[\partial(P, e) / \partial(v, T)] /(\partial P / \partial v)_{T} \\
& (\partial e / \partial P)_{s}=-P(\partial v / \partial P)_{s} \\
& (\partial e / \partial s)_{P}=T-P(\partial v / \partial s)_{P}
\end{aligned}
$$




\section{Temperature-Equilibrium Case.}

This section describes the case in which the mixture constituents relax from their initial conditions to equilibrium values of pressure $P$ and temperature $T$ while preserving the initial total volume $V$ and energy $E$. Following the MIXPAC procedure, ${ }^{5}$ a Newton-Raphson iteration is presented for calculating the changes in the EOS quantities needed to achieve the final equilibrium state. At the beginning of the $i$-th iteration, let the values of the EOS quantities for the $j$-th constituent be $v_{j}^{(i)}, e_{j}^{(i)}, s_{j}^{(i)}, P_{j}^{(i)}, T_{j}^{(i)}$. The two independent variables are chosen to be $(v, e)$, so

$$
s_{j}^{(i)}=s_{j}\left(v_{j}^{(i)}, e_{j}^{(i)}\right), \quad P_{j}^{(i)}=P_{j}\left(v_{j}^{(i)}, e_{j}^{(i)}\right), T_{j}^{(i)}=T_{j}\left(v_{j}^{(i)}, e_{j}^{(i)}\right)
$$

and the first partial derivatives of these quantities are also evaluated at $\left(v_{j}^{(i)}, e_{j}^{(i)}\right)$. Throughout the remainder of this section, the iteration superscript index $i$ will be suppressed, and changes in the EOS quantities during the iteration will be denoted by the symbol $\delta$.

The Newton-Raphson approximation uses first-order Taylor-series expansions to relate changes in the EOS quantities:

$$
\begin{aligned}
& \delta P_{j} \equiv P-P_{j} \equiv \delta v_{j}(\partial P / \partial v)_{e j}+\delta e_{j}(\partial P / \partial e)_{v j} \\
& \delta T_{j} \equiv T-T_{j} \equiv \delta v_{j}(\partial T / \partial v)_{e j}+\delta e_{j}(\partial T / \partial e)_{v j}
\end{aligned}
$$

These relations can be inverted to give

$$
\begin{aligned}
& \delta v_{j}=\left(P-P_{j}\right)(\partial v / \partial P)_{T j}+\left(T-T_{j}\right)(\partial v / \partial T)_{P j}, \\
& \delta e_{j}=\left(P-P_{j}\right)(\partial e / \partial P)_{T j}+\left(T-T_{j}\right)(\partial e / \partial T)_{P j}
\end{aligned}
$$

where expressions for the partial derivatives are given in Section 2. Note that a consistent first-order approximation for the change in specific entropy during the iteration is

$$
\delta s_{j}=\left(P_{j} / T_{j}\right) \delta v_{j}+\left(1 / \dot{T}_{j}\right) \delta e_{j}=\left(P-P_{j}\right)(\partial s / \partial P)_{T j}+\left(T-T_{j}\right)(\partial s / \partial T)_{P j}
$$

The constraints that the total volume and energy be preserved during the iteration then produce two linear equations for the two unknowns $P$ and $T$ :

$$
\begin{aligned}
& 0 \equiv \sum_{j} M_{j} \delta v_{j}=A_{v} P+B_{v} T-C_{v} \\
& 0 \equiv \sum_{j} M_{j} \delta e_{j}=A_{e} P+B_{e} T-C_{e} \\
& A_{v} \equiv \sum_{j} M_{j}(\partial v / \partial P)_{T j}, \quad B_{v} \equiv \sum_{j} M_{j}(\partial v / \partial T)_{P j} \\
& C_{v} \equiv \sum_{j} M_{j}\left[P_{j}(\partial v / \partial P)_{T j}+T_{j}(\partial v / \partial T)_{P j}\right]
\end{aligned}
$$




$$
\begin{aligned}
& A_{e} \equiv \sum_{j} M_{j}(\partial e / \partial P)_{T j}, \quad B_{e} \equiv \sum_{j} M_{j}(\partial e / \partial T)_{P j} \\
& C_{e} \equiv \sum_{j} M_{j}\left[P_{j}(\partial e / \partial P)_{T j}+T_{j}(\partial e / \partial T)_{P j}\right]
\end{aligned}
$$

The solutions of Eqs. (27) \& (28) give the temperature-equilibrium values:

$$
\begin{aligned}
& P=P^{E q} \equiv\left(C_{v} B_{e}-B_{v} C_{e}\right) /\left(A_{v} B_{e}-B_{v} A_{e}\right), \\
& T=T^{E q} \equiv\left(A_{v} C_{e}-C_{v} A_{e}\right) /\left(A_{v} B_{e}-B_{v} A_{e}\right) .
\end{aligned}
$$

If these new values of $P$ and $T$ are sufficiently close to the previous values, then the iteration has converged and can be terminated, and the final values of the EOS quantities for the mixture constituents are $v_{j}, e_{j}, s_{j}, P, T$. Otherwise, the new values of $P$ and $T$ are substituted into Eqs. (24) \& (25), and the results are used to increment $v_{j}$ and $e_{j}$ to begin the next iteration. As with any Newton-Raphson iteration, the convergence of this procedure requires that the initial values be sufficiently close to the final equilibrium values. Since such accurate initial values cannot be guaranteed, the changes in $v_{j}$ and $e_{j}$ are limited to prevent the Newton-Raphson iteration from diverging. Thus the new values to be used for the next iteration are $v_{j}+\mu_{v} \delta v_{j}$ and $e_{j}+\mu_{e} \delta e_{j}$, where expressions for the limit factors $\mu_{v}$ and $\mu_{e}$, which are both $\in(0,1]$, are given in the Appendix.

\section{Isentropic Case.}

This section describes the case in which the mixture constituents relax from their initial conditions to an equilibrium value of pressure $P$ while preserving their initial specific entropies as well as the initial total volume $V$ and energy $E$. In parallel with Section 3, a Newton-Raphson iteration is presented for calculating the changes in the EOS quantities needed to achieve the final equilibrium state. At the beginning of the $i$-th iteration, let the values of the EOS quantities for the $j$-th constituent be $v_{j}^{(i)}, e_{j}^{(i)}, s_{j}^{(i)}, P_{j}^{(i)}, T_{j}^{(i)}$. The two independent variables are chosen to be $(v, e)$, so

$$
s_{j}^{(i)}=s_{j}\left(v_{j}^{(i)}, e_{j}^{(i)}\right), \quad P_{j}^{(i)}=P_{j}\left(v_{j}^{(i)}, e_{j}^{(i)}\right), T_{j}^{(i)}=T_{j}\left(v_{j}^{(i)}, e_{j}^{(i)}\right)
$$

and the first partial derivatives of these quantities are also evaluated at $\left(v_{j}^{(i)}, e_{j}^{(i)}\right)$. Throughout the remainder of this section, the iteration superscript index $i$ will be suppressed, and changes in the EOS quantities during the iteration will be denoted by the symbol $\delta$.

The Newton-Raphson approximation uses first-order Taylor-series expansions to relate changes in the EOS quantities:

$$
\begin{aligned}
& \delta P_{j} \equiv P-P_{j} \equiv \delta v_{j}(\partial P / \partial v)_{s j}+\delta s_{j}(\partial P / \partial s)_{v j} \\
& \delta s_{j} \equiv\left(P / T_{j}\right) \delta v_{j}+\left(1 / T_{j}\right) \delta e_{j} \equiv 0
\end{aligned}
$$

These relations can be inverted to give

$$
\delta v_{j}=\left(P-P_{j}\right)(\partial v / \partial P)_{s j}
$$




$$
\delta e_{j}=-P \delta v_{j}
$$

where expressions for the partial derivatives are given in Section 2.

Since $P$ was used instead of $P_{j}$ in Eqs. (37) \& (39), the constraints that the total volume and energy be preserved during the iteration produce a single linear equation for the unknown $P$ :

$$
\begin{aligned}
& 0 \equiv \sum_{j} M_{j} \delta v_{j} \equiv-\sum_{j} M_{j} \delta e_{j} / P \equiv A P-C \\
& A \equiv \sum_{j} M_{j}(\partial v / \partial P)_{s j}, \quad C \equiv \sum_{j} M_{j} P_{j}(\partial v / \partial P)_{s j}
\end{aligned}
$$

The solution of Eq. (40) gives the isentropic value:

$$
P=P^{I s} \equiv C / A \text {. }
$$

If this new value of $P$ is sufficiently close to the previous value, then the iteration has converged and can be terminated, and the final values of the EOS quantities for the mixture constituents are $v_{j}, e_{j}, s_{j}, P, T_{j}$. Otherwise, the new value of $P$ is substituted into Eqs. (38) \& (39), and the results are used to increment $v_{j}$ and $e_{j}$ to begin the next iteration. As with any Newton-Raphson iteration, the convergence of this procedure requires that the initial values be sufficiently close to the final equilibrium values. Since such accurate initial values cannot be guaranteed, the changes in $v_{j}$ and $e_{j}$ are limited to prevent the Newton-Raphson iteration from diverging. Thus the new values to be used for the next iteration are $v_{j}+\mu_{v} \delta v_{j}$ and $e_{j}+\mu_{e} \delta e_{j}$, where expressions for the limit factors $\mu_{v}$ and $\mu_{e}$, which are both $\in(0,1]$, are given in the Appendix.

\section{Generalized Procedure.}

This section describes a generalized procedure for calculating the EOS of a material mixture whose constituents relax from their initial conditions to an equilibrium value of pressure $P$ while preserving the initial total volume $V$ and energy $E$. The strategy is to choose the changes in the EOS quantities to be fixed (but arbitrary) linear combinations of the corresponding changes in the two extremes of temperature equilibrium and isentropy. In parallel with Sections 3 \& 4, a Newton-Raphson iteration is presented for calculating the changes in the EOS quantities needed to achieve the final equilibrium state. At the beginning of the $i$-th iteration, let the values of the EOS quantities for the $j$-th constituent be $v_{j}^{(i)}, e_{j}^{(i)}, s_{j}^{(i)}, P_{j}^{(i)}, T_{j}^{(i)}$. The two independent variables are chosen to be $(v, e)$, so

$$
s_{j}^{(i)}=s_{j}\left(v_{j}^{(i)}, e_{j}^{(i)}\right), \quad P_{j}^{(i)}=P_{j}\left(v_{j}^{(i)}, e_{j}^{(i)}\right), \quad T_{j}^{(i)}=T_{j}\left(v_{j}^{(i)}, e_{j}^{(i)}\right),
$$

and the first partial derivatives of these quantities are also evaluated at $\left(v_{j}^{(i)}, e_{j}^{(i)}\right)$. Throughout the remainder of this section, the iteration superscript index $i$ will be suppressed, and changes in the EOS quantities during the iteration will be denoted by the symbol $\delta$. Furthermore, the superscript $E q$ will denote temperature-equilibrium values given in Section 3, and the superscript $I s$ will denote isentropic values given in Section 4. 
The Newton-Raphson approximation uses first-order Taylor-series expansions to relate changes in the EOS quantities:

$$
\begin{aligned}
& \delta P_{j} \equiv P-P_{j} \equiv \alpha \delta P_{j}^{E q}+(1-\alpha) \delta P_{j}^{I s} \equiv \delta v_{j}(\partial P / \partial v)_{s j}+\delta s_{j}(\partial P / \partial s)_{v j} \\
& \delta s_{j} \equiv \alpha \delta s_{j}^{E q} \equiv\left(1 / T_{j}\right)\left[\alpha P_{j} \delta v_{j}^{E q}+(1-\alpha) P^{I s} \delta v_{j}^{I s}+\delta e_{j}\right]
\end{aligned}
$$

where $\alpha \in[0,1]$ is the fixed (but arbitrary) fractional contribution from the temperature-equilibrium solutions, which depends on the degree of contact of the mixture constituents. These relations can be inverted to give

$$
\begin{aligned}
& \delta v_{j}=\left(P-P_{j}\right)(\partial v / \partial P)_{s j}+\delta s_{j}(\partial v / \partial s)_{P j}=\alpha \delta v_{j}^{E q}+(1-\alpha) \delta v_{j}^{I s} \\
& \delta e_{j}=\alpha\left(-P_{j} \delta v_{j}^{E q}+T_{j} \delta s_{j}^{E q}\right)-(1-\alpha) P^{I s} \delta v_{j}^{I s}=\alpha \delta e_{j}^{E q}+(1-\alpha) \delta e_{j}^{I s},
\end{aligned}
$$

where expressions for the partial derivatives are given in Section 2.

The constraints that the total volume and energy be preserved during the iteration are automatically satisfied since they are satisfied separately by the temperature-equilibrium and isentropic contributions:

$$
\sum_{j} M_{j} \delta v_{j}=0, \quad \sum_{j} M_{j} \delta e_{j}=0
$$

Eqs. (33), (34), \& (42) are used to calculate new values of $P^{E q}, T^{E q}$, and $P^{I s}$, and the generalized equilibrium pressure is then

$$
P=\alpha P^{E q}+(1-\alpha) P^{I s}
$$

If this new value of $P$ is sufficiently close to the previous value, then the iteration has converged and can be terminated, and the final values of the EOS quantities for the mixture constituents are $v_{j}, e_{j}, s_{j}, P, T_{j}$. Otherwise, the new values of $P^{E q}, T^{E q}$, and $P^{I s}$ are substituted into Eqs. (24); (25), (38), \& (39) to calculate new values of $\delta v_{j}^{E q}, \delta e_{j}^{E q}, \delta v_{j}^{I s}$, and $\delta e_{j}{ }^{I s}$. These, in turn, are substituted into Eqs. (46) \& (47), and the results are used to increment $v_{j}$ and $e_{j}$ to begin the next iteration. As with any Newton-Raphson iteration, the convergence of this procedure requires that the initial values be sufficiently close to the final equilibrium values. Since such accurate initial values cannot be guaranteed, the changes in $v_{j}$ and $e_{j}$ are limited to prevent the Newton-Raphson iteration from diverging. Thus the new values to be used for the next iteration are $v_{j}+\mu_{v} \delta v_{j}$ and $e_{j}+\mu_{e} \delta e_{j}$, where expressions for the limit factors $\mu_{v}$ and $\mu_{e}$, which are both $\in(0,1]$, are given in the Appendix. 


\section{Conclusions.}

This report has described a procedure for calculating the EOS of a material mixture whose constituents are in pressure equilibrium but not necessarily in temperature equilibrium. The procedure is a generalization of the MIXPAC procedure ${ }^{5}$ for calculating the EOS of a material mixture whose constituents are in both pressure and temperature equilibrium. Since the strategy is to choose the changes in the EOS quantities to be fixed (but arbitrary) linear combinations of the corresponding changes in the two extremes of temperature equilibrium and isentropy, those two extremes can be reproduced as special cases. All EOS quantities are considered to be functions of the specific volume $v$ and energy $e$, and a Newton-Raphson iteration (modified to prevent it from diverging) is used to calculate the changes in these quantities needed to achieve the final equilibrium state. The procedure is thus well suited for using the EOSPAC subroutine package ${ }^{4}$ to interpolate the tabular EOS functions contained in the Sesame EOS Data Library ${ }^{2,3}$ at LANL.

\section{Acknowledgment.}

This work was performed under the auspices of the U.S. Department of Energy by the Los Alamos National Laboratory under contract number W-7405-ENG-36.

\section{References.}

1. G. Dahlquist and A. Bjorck, Numerical Methods, translated by N. Anderson, Prentice-Hall, Inc., Englewood Cliffs (1974), ch. 6.

2. "An Invitation to Participate in the LASL Equation of State Library," edited by N.G. Cooper, Los Alamos Scientific Laboratory report LASL-79-62 (February 1980).

3. "SESAME '83: Report on the Los Alamos Equation-of-State Library," Los Alamos National Laboratory report LALP-83-4 (February 1983).

4. C.W. Cranfill, "EOSPAC: A Subroutine Package for Accessing the Los Alamos Sesame EOS Data Library," Los Alamos National Laboratory report LA-9728-M (August 1983).

5. C.W. Cranfill, "MIXPAC: A Subroutine Package for Calculating the Equations of State for Equilibrium Mixtures of Materials," Los Alamos National Laboratory report LA-9861-M (August 1983). 


\section{Appendix: Preventing the Newton-Raphson Iteration from Diverging.}

It is well known that a Newton-Raphson iteration generally has a finite radius of convergence and hence will converge only if the initial guess is sufficiently close to the final solution. ${ }^{1}$ If such an accurate initial guess cannot be guaranteed, a modification is required to prevent the iteration from diverging. For example, when a Newton-Raphson iteration fails to converge, it can be supplemented or replaced by a more robust (but less efficient) iteration - e.g. either successive-bisection or regulafalsi iteration ${ }^{1}$ is guaranteed to converge if two successive guesses that bracket the true solution can be found. The more robust iteration can be used either to achieve the final convergence or to improve the initial guess for the Newton-Raphson iteration. Unfortunately, for a multivariate iteration such as is needed in the present application to balance the partial pressures in a material mixture, it is difficult or impossible to find two successive guesses that bracket the true solution for all the variables. Therefore, an alternate modification is adopted here to prevent the NewtonRaphson iteration from diverging - the changes in the independent variables during each iteration are limited.

Following the notation in Sections 3-5, let $v_{j}$ and $e_{j}$ be the values of the independent variables at the beginning of an iteration, and let $\delta v_{j}$ and $\delta e_{j}$ be the Newton-Raphson guesses for the changes in these values, then the new values to be used for the next iteration are taken to be $v_{j}+\mu_{v} \delta v_{j}$ and $e_{j}+\mu_{e} \delta e_{j}$, where the limit factors $\mu_{v}$ and $\mu_{e}$ are both $\in(0,1]$. To derive expressions for the limit factors, account must first be taken of the physical bounds on the independent variables:

$$
0<v_{j}+\mu_{v} \delta v_{j}<V / M_{j}, \quad 0<e_{j}+\mu_{e} \delta e_{j}<E / M_{j}
$$

These inequalities can be manipulated to give bounds on the changes to the independent variables:

$$
-v_{j}<\mu_{v} \delta v_{j}<v_{j}^{*} \equiv V / M_{j}-v_{j}, \quad-e_{j}<\mu_{e} \delta e_{j}<e_{j}^{*} \equiv E / M_{j}-e_{j}
$$

These bounds are too loose, however, since the Newton-Raphson guesses can oscillate between them indefinitely without ever converging. Thus additional factors $\phi_{v}$ and $\phi_{e}$, both $\in(0,1)$, are introduced to reduce the allowed changes in the independent variables:

$$
-\phi_{v} v_{j} \leq \mu_{v} \delta v_{j} \leq \phi_{v} v_{j}^{*}, \quad-\phi_{e} e_{j} \leq \mu_{e} \delta e_{j} \leq \phi_{e} e_{j}^{*}
$$

The corresponding expressions for the limit factors are then

$$
\mu_{v} \equiv \min _{j}\left[1, \phi_{v} \max \left(-v_{j} / \delta v_{j}, v_{j}^{*} / \delta v_{j}\right)\right], \quad \mu_{e} \equiv \min _{j}\left[1, \phi_{e} \max \left(-e_{j} / \delta e_{j}, e_{j}^{*} / \delta e_{j}\right)\right]
$$

A reasonable first choice for the fractions $\phi_{v}$ and $\phi_{e}$ is 0.5 , and if the iteration still fails to converge, a smaller value can be tried.

This modification prevents the Newton-Raphson iteration from diverging to unphysical values of the independent variables, and while the resulting procedure is not guaranteed to converge, it seems to do so in most cases. 